\section{Phase III: Arsentrioxid bei APL}

Das Onkoprotein PML-RARA ist charakteristisch für die akute Promyelozytenleukämie (APL), die auf Arsentrioxid anzusprechen scheint. In einer Phase-IIIStudie wurde daher die Kombination Arsentrioxid plus All-trans-Retinsäure (ATRA) dem Standardregime Idarubicin plus ATRA gegenübergestellt.

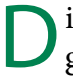
ie APL ist eine chemosensitive Subgruppe der AML, charakterisiert durch das PML-RARA-Fusionsprotein. Verschiedenen Studien zufolge scheint Arsentrioxid zu einer Reduktion von PML-RARA zu führen. In der AML17Studie wurde nun die Kombination ATRA plus Arsentrioxid bei Hoch- und Niedrigrisiko-Patienten ( $\geq 16$ Jahre) geprüft. Zwischen Mai 2009 und Oktober 2013 wurden 235 Patienten in die Studie aufgenommen (im Mittel 47 Jahre; 57 Hochrisiko-Patienten). 1:1 randomisiert

Alopezie ist häufig unter ATRA/Idarubicin.

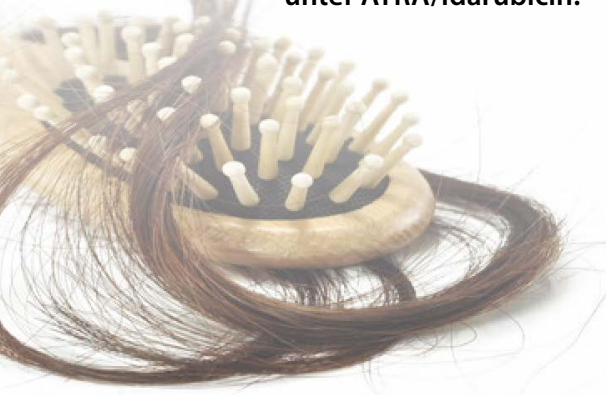

bekamen sie entweder ATRA plus Arsentrioxid oder ATRA plus Idarubicin. Hochrisiko-Patienten $\left(>10 \times 10^{9}\right.$ Leukozyten/l) konnte eine initiale Dosis des Immunkonjugats Gemtuzumab-Ozogamicin $\left(6 \mathrm{mg} / \mathrm{m}^{2}\right.$ i.v.) verordnet werden. Die Patienten erhielten weder eine Erhaltungstherapie noch eine ZNS-Prophylaxe. Primärer Endpunkt dieser Phase-III-Studie war die Lebensqualität.

In der ATRA/Arsentrioxid-Gruppe erreichten mehr Patienten eine molekular gesicherte Komplettremission als in der ATRA/Idarubicin-Gruppe ( 91 vs. $88 \%$ ). Das Gesamtüberleben war nach 4 Jahren nicht signifikant verschieden. Allerdings war die Rate für das rückfallsfreie 4-Jahres-Überleben der Patienten mit molekular bestätigter Komplettremission in der ATRA/Arsentrioxid-Gruppe deutlich höher (98 vs. $70 \%$; $\mathrm{p}<0,0001$ ).

Die Lebensqualität, gemessen anhand des EORTC-QLQ-C30-Fragebogens und der HADS (Hospital Anxiety and Depression Scale) unterschied sich in den Gruppen nicht signifikant. Insgesamt berichteten 57 Patienten in der Kontroll- und 40 Patienten in der Arsentrioxid-Gruppe von Nebenwirkungen des Grads 3/4. Eine Alopezie nach dem ersten Behandlungszyklus entwickelten $23 \%$ der Patienten in der IdarubicinGruppe, aber nur 5\% der Patienten, die mit Arsentrioxid behandelt wurden. Erhöhte Werte der Alanin-Aminotransferase (ALT) traten häufiger unter ATRA/Arsentrioxid auf (25 vs. $10 \%$ ) und orale Toxizität öfter unter ATRA/Idarubicin (19 vs. $1 \%)$. Andere Toxizitäten erreichten die $10 \%$-Marke nicht. Patienten in der ATRA/Arsentrioxid-Gruppe benötigten signifikant seltener Supportivbehandlungen.

Fazit: ATRA plus Arsentrioxid ist eine Option bei APL-Patienten mit niedrigem oder auch hohem Risiko. Die Heilungsrate ist mit der Heilungsrate unter der Standardtherapie vergleichbar und die Rezidivrate niedriger. Trotz leicht unterschiedlicher Toxizitätsprofile war die Lebensqualität in den Gruppen vergleichbar.

Christina Berndt

Burnett AK et al. Arsenic trioxide and all-trans retinoic acid treatment for acute promyelocytic leukaemia in all risk groups (AML17): results of a randomised, controlled, phase 3 trial. Lancet Oncol. 2015;16(13):1295-305.

\title{
ALL: Veränderungen der Kopienzahl beeinflussen Prognose
}

Welcher Zusammenhang besteht zwischen Veränderungen der Kopienzahl bestimmter Gene und dem Gesamtüberleben bei akuter lymphoblastischer Leukämie (ALL)?

D ie Prognose einer ALL hängt von $\mathrm{Al}$ ter, Leukozytenzahl, genetischen Faktoren und v. a. dem Ansprechen auf die Therapie ab. Veränderungen der Kopienzahl bestimmter Gene scheinen mit einer ungünstigen Prognose assoziiert zu sein, aber die Daten dazu sind widersprüchlich. Die spanische PETHEMAGruppe untersuchte nun Häufigkeit und prognostischen Einfluss von Veränderungen der Kopienzahl in 12 Genregionen bei 142 Jugendlichen und Erwach- senen mit De-novo-Precursor-B-ZellALL.

Eine Deletion von CDKN2A/B war die häufigste Veränderung der Kopienzahl (42\%), gefolgt von IKZF1-Deletionen (35\%). Letztere kamen häufiger bei Philadelphia-Chromosom(Ph)-positiver ALL vor als bei Ph-negativer ALL (52 vs. $30 \%)$. Außerdem waren IKZF1-Deletionen mit einem höheren Alter und höheren Leukozytenzahlen assoziiert.

In der multivariaten Analyse waren ein höheres Alter und Deletionen von EBF1 mit weniger Komplettremissionen assoziiert (Hazard Ratio [HR] 0,949 bzw. 0,135; bei Ph-negativer ALL 0,946 bzw. 0,118 ). Hohe Leukozytenzahlen und fokale IKZF1-Deletionen korrelierten mit dem Rezidivrisiko (HR 1,005 bzw. 1,869). CDKN2A/B-Deletionen und höheres Alter beeinflussten das Gesamtüberleben negativ (HR 1,038 bzw. 2,545; Phnegative ALL: HR 1,044 bzw. 2,105).

Fazit: Bei Jugendlichen und Erwachsenen mit Precursor-B-Zell-ALL gehen Deletionen in den Genen EBF1, IKZF1 und $\mathrm{CDKN} 2 \mathrm{~A} / \mathrm{B}$ mit einer ungünstigen Prognose einher. Diese Veränderungen der Kopienzahlen sollten in die initiale Risikobeurteilung einfließen. Judith Neumaier

Ribera J et al. Prognostic significance of copy number alterations in adolescent and adult patients with precursor B acute lymphoblastic leukemia enrolled in PETHEMA protocols. Cancer. 2015;121(21):3809-17. 